\title{
Bibliometría brasileira: la difusión de su literatura
}

\author{
Rubén Urbizagástegui Alvarado \\ University of California at Riverside, Library, Riverside, CA, USA \\ ruben@ucrl.edu \\ Cristina RestrepoArango \\ Universidad de Córdoba, División de Bibliotecas y Recursos Educativos, Montería, Córdoba, Colombia \\ crestrepoarango@gmail.com
}

DOI: https://doi.org/10.26512/rici.v13.n1.2020.27922

Recebido/Recibido/Received: 2019-10-24

Aceitado/Aceptado/Accepted: 2020-01-18

Resumen: Analiza la difusión de la literatura sobre las "metrías" (bibliometría, cienciometría, infometría, webmetría, archivometría, tecnometría, patentometría, etc.) producida y publicada en el Brasil y por brasileros en el exterior. Para analizar la difusión de esta literatura se hace uso del modelo de difusión de innovaciones de Bass, toda vez que la literatura no deja de ser también una innovación o de comunicar una innovación. Fueron identificados 6170 documentos producidos por 9.707 autores diferentes desde 1973 hasta 2018. El modelo el modelo de difusión de las innovaciones de Bass estimó que la influencia que los investigadores reciben de la lectura de otros documentos sobre este asunto en los sistemas de información es menor al 1\% (coeficiente de innovación). El coeficiente de imitación que mide la influencia que un grupo de adoptantes ejerce sobre las decisiones de adopción de otros potenciales adoptantes es igual al $22 \%$. El parámetro que estima el punto de saturación del campo predice que este se alcanzará cuando se hayan publicado 165450 documentos.

Palabras clave: Modelo de Bass. bibliometría. cienciometría. Brasil. difusión de innovaciones.

\section{Bibliometria brasileira: a difusão de sua literatura}

Resumo: Analisa a difusão da literatura sobre as "metrias" (bibliometria, cienciometria, infometria, webometria, arquivometria, tecnometria, patentometria, etc.) produzidas e publicadas no Brasil e por brasileiros no exterior. Para analisar a difusão dessa literatura, utiliza-se o modelo de difusão de inovações de Bass, uma vez que a literatura pode ser considerada uma inovação ou serve para comunicar uma inovação. 6170 documentos produzidos por 9707 autores diferentes foram identificados entre 1973 e 2018 . O modelo de difusão das inovações de Bass, estimou que a influência que os pesquisadores recebem da leitura de outros documentos sobre esse assunto em sistemas de informação é menor que $1 \%$ (coeficiente de inovação). 0 coeficiente de imitação que mede a influência que um grupo de adotantes exerce sobre as decisões de adoção de outros adotantes potenciais é igual a $22 \%$. O parâmetro que estima o ponto de saturação do campo prevê que isso será alcançado quando forem publicados 165450 documentos.

Palavras chave: Modelo de Bass. bibliometria. cienciometria. Brasil. difusão de inovações.

\section{Brazilian bibliometrics: diffusion of its literature}

Abstract: It analyzes the diffusion of literature on "metrics" (bibliometrics, scientometrics, infometrics, webmetrics, archivemetrics, technometrics, patentmetrics, etc.) produced and published in Brazil and by Brazilians abroad. In order to analyze the diffusion of this literature, the Bass Model of Innovations was used, since the literature is also a kind of an innovation. From 1973 to 2018, a total of 6170 documents produced by 9707 different authors, was identified. The Bass model of innovations estimated that the influence 
researchers receive from reading other documents on this subject in information systems is less than $1 \%$ (innovation coefficient). The coefficient of imitation that measures the influence that a group of adopters exerts on the adoption decisions of other potential adopters is equal to $22 \%$. The parameter that estimates the saturation point of the field, predicts that this will be reached when 165450 documents have been published.

Keywords: Bass model. bibliometrics. scientometrics. diffusion innovation. Brazil.

\section{Introducción}

El esfuerzo individual de los científicos es la producción de nueva información, ya sea con la formulación de nuevos conceptos, descripción de nuevos datos o integración de los datos conceptuales en nuevas investigaciones. Para que esas formulaciones sean exitosas contribuciones a la ciencia deben ser comunicadas de tal forma, que puedan ser comprendidas y verificadas por otros científicos y después utilizadas para proporcionar nuevas bases para mayores exploraciones (GARVEY, 1979, GARVEY; GRIFFITH, 1979). Para realizar nuevas investigaciones los científicos se basan en investigaciones y teorías realizadas por sus predecesores, por eso se afirma que "[...]lo nuevo es construido sobre lo viejo y, por lo tanto, depende en cierto grado del acceso a lo viejo" (LESSIG, 2001, p. 105). Isaac Newton en una carta a Robert Hooke en 1676, resaltaba indirectamente la importancia del acceso a descubrimientos anteriores al afirmar que "[...]si vi un poquito más fue porque me apoyé en los hombros de gigantes" (NEWTON, 1676).

Ése ver un poquito más apoyado sobre los hombros de gigantes, en otras palabras, es el reconocimiento de la comunicación de las ideas realizadas por otros, por medio de los impresos escritos y publicados por sus antecesores y la forma en que cada generación se ha apoyado en el conocimiento y el saber de las generaciones precedentes. La historia de la ciencia tiene como un hilo conductor la influencia de sus predecesores, la repercusión en sus contemporáneos y en las generaciones posteriores, así "Copérnico, padre de la teoría heliocéntrica, se basó en filósofos griegos como Hiparco y Ptolomeo, cuyas obras fueron preservadas en los países islámicos durante los siglos IX a XIII. La obra de Copérnico fue clave para que décadas más tarde Galileo y Kepler pusieran los cimientos de la revolución científica que culminó con la obra de Newton" (LOZANOMEJÍA, 1988). Este reconocimiento implica que existe alguna forma de relación entre la ciencia y los documentos publicados, pues, si los documentos no se publican no existiría la difusión de la ciencia. Los científicos ganan membresía tribal al publicar los resultados de sus investigaciones. Esa acción de publicación tiene la función de hacer públicos los resultados de las investigaciones, mostrar los acuerdos o desacuerdos con publicaciones anteriores, llamar la atención para eventuales olvidos o errores, o en algunos casos, simplemente la reproducción y/o aplicación de los modelos o técnicas estudiadas. La función del científico es producir y publicar trabajos originales, comunicarlos a sus pares y contribuir al conocimiento público (ZIMAN, 1969), pero para constituirse en avance del 
conocimiento, esa publicación debe ser novedosa en su concepción, en su metodología, en los pormenores descritos y en sus resultados (WATERMAN, 1966). La ciencia se caracteriza por ser de conocimiento público y la literatura producida sobre un asunto científico es tan importante cuanto la propia investigación que la incorpora, pues, los artículos científicos son derivados de los anteriores y en gran medida poco originales, porque precisamente se basan investigaciones anteriores. "La evidencia de esto es evidente en la larga lista de citas que siempre deben publicarse con cada nueva contribución. Estas citas no solo avalan la autoridad y relevancia de las declaraciones que deben respaldar; sino que también integran todo el trabajo en un contexto de logros anteriores y aspiraciones actuales" (ZIMAN, 1969, p. 318).

Quiere decir entonces que la cientificidad de la investigación no descansa solamente en su ejecución, sino también en su publicación. Por ejemplo, Pierce (1990, p. 55) argumenta que la investigación científica es reconocible como tal, no solo por las condiciones en la que es producida, sino por la manera en la que es presentada y publicada hasta el punto de que "la ciencia no existiría si los resultados de los trabajos científicos no fuesen comunicados" (HO et al., 2003, p. 369). Se entiende, entonces, que la actividad científica debe materializarse en documentos escritos validados y legitimados por la comunidad científica. Esos documentos se constituyen, a su vez, en importantes indicadores del estado de desarrollo de un campo, es decir, la literatura producida se convierte en una especie de archivo histórico de la ciencia y es esencial para el mantenimiento de un registro público permanente de los resultados, observaciones, cálculos, teorías, etc. de la ciencia y sirven como base para posteriores referencias de otros científicos. También sirven de base para el análisis del desarrollo de la ciencia, de una disciplina, sub-disciplina o campo científico. Cuando a esas publicaciones se las trata como "átomos de conocimiento, se ha sido capaz de derivar un modelo que muestra cómo los nuevos artículos están relacionados con los viejos artículos" (PRICE, 1969) produciendo así razonables pruebas de la validad del conteo de los documentos como una legítima medida de su producción. Por eso es que se afirma que el viejo conocimiento da lugar a un nuevo conocimiento y que la ciencia crece de una manera estructurada desde la epidermis (la literatura producida) hasta su estado actual, en que la literatura publicada se "convierte en una buena indicación (indicador) de la ciencia simplemente porque la alta motivación para la publicación existe, porque la publicación es el único signo externo de que el trabajo ha sido realizado y que[el autor] puede entrar a formar parte del frente de investigación, producir nuevos conocimientos, y [...] que dé a su originador la inmortalidad de su trabajo en un archivo perpetuo" (PRICE, 1969, p. 95) de la ciencia producida. Por eso se asevera que el conocimiento científico crece de la misma forma que crece cualquier cuerpo viviente muy similar al crecimiento de los organismos y que en ese proceso de crecimiento la información es la materia prima, la precursora del conocimiento de modo que "el conocimiento emerge de la destilación, la formalización e integración de la materia 
prima en conceptos y reglas" (WEISS, 1960, p. 1717). En otras palabras, conforme crece el conocimiento, también crece la literatura que la explicita, por lo tanto, se hace necesario estudiar la forma de difusión de esta literatura.

A pesar de que existen muchos estudios sobre la difusión de las innovaciones y la difusión de las tecnologías, cuyos comportamientos parecen ser similares a la difusión de la literatura publicada, en el campo de la bibliotecología y la ciencia de la información latinoamericana no existen estudios referentes a la difusión de la literatura publicada como una forma de innovación. Para dar cuenta de la difusión de las innovaciones existen varios modelos, pero el más utilizado es el modelo de Bass (1969). Hasta donde es del conocimiento de los autores de este artículo apenas dos trabajos han hecho uso del modelo de Bass para estudiar el crecimiento de la literatura sobre investigaciones en laser (JAIN; GARG, 1992) y sobre la potencia solar (GARG et al., 1993). En América Latina merece destacar el artículo de Urbizagástegui-Alvarado (2017) referente a la literatura sobre Argopectenpurupuratus y de Restrepo-Arango (2018) sobre la literatura de los indígenas zenú en Colombia. Estos autores recomiendan mayores investigaciones utilizando bibliografías de campos científicos diferentes.

Por esa razón, el objetivo de este artículo es analizar la difusión de la literatura sobre las "metrías "en el Brasil utilizando el modelo de difusión de innovaciones de Bass (1969), es decir, analizar la literatura publicada sobre bibliometría, cienciometría, infometría, webmetría, archivometría, tecnometría, patentometría, etc., producidas y publicada sen el Brasil y por brasileros en el exterior, ya que la literatura académica o científica también es una forma de comunicar una innovación; en este caso las técnicas bibliométricas producidas en el Brasil.

Para lograre objetivo propuesto, este artículo está estructurado de la forma siguiente: la introducción, que está delimitada por el asunto de la investigación, se caracteriza el problema y se establece el objetivo. El marco teórico y revisión de la literatura donde son explorados los conceptos de difusión e innovación como constructos teóricos centrales a esta investigación. Luego vienen los procedimientos metodológicos adoptados (población estudiada, recolección de los datos y forma de medición de los datos recogidos). Después el análisis de los resultados obtenidos y las conclusiones y recomendaciones. Finalmente, la lista de las referencias bibliográficas utilizadas en esta investigación.

\section{Marco teórico y revisión de la literatura}

Una innovación es un cambio que introduce novedades en un espacio social. Es la transformación del conocimiento en nuevos productos y servicios. Por ejemplo, las ideas solo pueden resultar en innovaciones luego que estas son implementadas como nuevas técnicas o procedimientos que se imponen y consolidan en un campo científico, por medio del proceso de 
difusión. Una innovación es el proceso de transformar las ideas en valores para las organizaciones y usuarios; esta se inicia con la generación de nuevas ideas, pasando por un tamizaje de viabilidad, hasta la implementación de un nuevo producto mejorado (GARCÍA GONZÁLEZ, 2012, GARZÓN; IBARRA, 2013). Una característica fundamental de la innovación es que ofrece soluciones a las necesidades no satisfechas de los usuarios, sobre todo de aquellas necesidades que no son conscientemente capaces de expresar como una necesidad no satisfecha, pero para que cualquier innovación llegue al mercado y genere consecuencias económicas directas, tiene que ser difundida. Por lo tanto, el proceso de difusión de las innovaciones es el mecanismo, por medio del cual una innovación es comunicada, es decir, es diseminada en un sistema social, por medio de un determinado canal o conjunto de canales de comunicación. La difusión de una innovación es un tipo especial de comunicación, ya que los mensajes se refieren a un nuevo tipo de ideas, tecnologías, modelos de pensamiento y similares para los miembros de un sistema social. Es decir, la difusión de la innovación es el proceso de introducción de nuevas técnicas de análisis de la literatura que son impulsadas por influencias sociales. En el caso de la bibliometría brasilera, la difusión de las técnicas bibliométricas se realiza, por medio de las revistas académicas publicadas en el campo de la ciencia de la información. Un primer acercamiento a este asunto fue hecho por Urbizagástegui-Alvarado (1984) y otros investigadores (MACHADO; PINTO, 2005, PINHEIRO; SILVA, 2008), pero con modelos diferentes.

Una corriente de importancia para los estudios de la difusión de las innovaciones procede de los estudios de la comunicación social. Estos estudios se centraron sobre las formas como los seres humanos toman sus decisiones y los factores que afectan esas decisiones, es decir, las influencias sociales que reciben al momento de tomar decisiones. Los estudios sobre los mecanismos de la difusión de las innovaciones inicialmente fueron llevados a cabo por Ryan y Gross (1943), dos sociólogos rurales que estudiaron la difusión de la semilla híbrida del maíz en dos comunidades del estado de lowa en los Estados Unidos. Posteriormente, en la década de los 50 realizaron investigaciones similares, pero centraron su interés en el poder de la comunicación informal como un complemento a las influencias de la comunicación de masas. Descubrieron que la comunicación informal está muy expandida en la sociedad y que ciertas personas eran más centrales e influyentes que otras dentro de un grupo social. Llamaron a este grupo "líderes de opinión" (WEIMANN, 1994). Aparentemente, los individuos interactúan con otros individuos para transmitir información, por ello se influencian mutuamente y esta influencia es mucho más poderosa de lo que hasta esa época se había reconocido. Esta comprobación llevó a establecer un modelo de comunicación en dos etapas: los líderes de opinión tienen la habilidad de difundir información, pues, están en una posición que les facilita la difusión de la información, ya que tienen acceso a una red 
de personas, por medio de las cuales difunden la información. Se concluye, entonces, que habilidad y acceso son las combinaciones adecuadas para ganar influencia y visibilidad.

Los primeros artículos que analizaron la difusión de las innovaciones comenzaron en los años 1960. Los primeros modelos fueron propuestos por Fourty Woodlock (1960) quienes asumieron que el proceso de difusión estaba influenciado principalmente por los medios de comunicación de masas. También Mansfield (1961) asumió que el proceso estaba influenciado principalmente por la comunicación interpersonal (boca a boca). El objetivo del modelo de difusión es identificar, describir y representar el ciclo de vida de una innovación, especialmente su crecimiento y saturación (ROGERS, 1963). Una innovación no crece desde el instante de su lanzamiento, sino que su evolución consiste en cuatro etapas: (a) una etapa de introducción de lento crecimiento; (b) una etapa de más rápido crecimiento; (c) una etapa de madurez que se caracteriza típicamente por poco o ningún crecimiento, y (d) una última etapa de saturación y declive donde la innovación cae debido a la obsolescencia o sustitución por otras innovaciones más ventajosas (MAHAJAN et al., 1990, ROGERS, 1963). Sin embargo, el análisis de Rogers es descriptivo, no ofrece ningún modelo matemático o ecuación para obtener una predicción adecuada de la adopción de las innovaciones en un caso específico, pero describe el proceso de adopción con profusión de detalles. Esta teoría de las innovaciones de Rogers casi no ha sido explorada. En el campo de la bibliotecología y ciencia de la información solo se conoce el estudio de Pérez Pulido y Terrón Torrado (2004) aplicándolo a la adopción de recursos electrónicos por la Universidad de Extremadura en España. El otro estudio es el de Kapoor et al. (2011), quienes analizaron las citas al libro de Rogers (1962) de 1996 a 2001.

Estos estudios sirvieron de base para que Frank Bass (1969) desarrollara un modelo de difusión matemático que incorporó las propuestas anteriores. Para este autor la difusión de una innovación es influenciada por dos procesos: los medios de comunicación de masas y la comunicación interpersonal. El modelo es utilizado para predecir el comportamiento de las innovaciones y nuevas tecnologías bajo dos condiciones: (a) la innovación o tecnología ha sido recientemente introducida en el mercado y los introductores han observado su comportamiento de adopción durante cierto período de tiempo; o (b) la innovación aún no ha sido introducida en el mercado pero es probable que su comportamiento de adopción sea muy similar con el de algunas innovaciones o tecnologías ya existentes y cuyo patrón de adopción ya se conoce. El modelo intenta predecir cuántos actores o agentes finalmente adoptarán el nuevo producto, pues, es innegable que existe interacción entre los actores, lo que induce a ciertos agentes a imitar el comportamiento de los innovadores. La posición del innovador dentro de una red social puede afectar el patrón de difusión. 
Siguiendo esta propuesta se han desarrollado muchas investigaciones en diferentes campos del conocimiento. Por ejemplo, Min et al. (2018) reconocen que las citas en la literatura científica son importantes tanto para rastrear el desarrollo histórico de las ideas científicas como para pronosticar las tendencias de investigación. Sin embargo, los mecanismos de difusión subyacentes al proceso de citas siguen siendo poco conocidos, a pesar del uso frecuente y prolongado de los recuentos de citas para fines de evaluación dentro de la comunidad científica. Extendieron el estudio de la dinámica de citas a un proceso de difusión más general para comprender cómo el crecimiento de las citas se asocia con diferentes patrones de difusión. Aplicaron el modelo de Bass y encontraron una relación positiva entre un patrón de "bajo p" y un "bajo q" con el alto impacto científico. Sin embargo, un pico de citación puntual producido por un cambio rápido en el recuento de citas tiene un efecto negativo en el impacto futuro. Además, sugirieron un indicador simple del nivel de saturación para estimar aproximadamente la etapa actual de un documento individual en el ciclo de vida, y su potencial para atraer atención futura. Ntwoku et al. (2017) afirmaron que en casi todos los países de bajos ingresos, las pequeñas y medianas empresas (PYME) son un motor principal para el desarrollo económico del país. Si bien existen muchos estudios de difusión de las tecnologías de la información y las comunicaciones (TIC), solo unos pocos se centran en países de bajos ingresos y aún menos emplean análisis basados en el modelo de Bass para examinar la difusión de las TIC en estos países. Este estudio aplica el modelo de difusión Bass para comprender la adopción de las TIC por parte de las PYME en Camerún, un país de bajos ingresos. Aplicaron el modelo de Bass encontraron que la difusión de las TIC entre las PYME en el contexto de una economía de bajos ingresos está impulsada en gran medida por fuerzas de imitación más que por fuerzas de innovación. Contribuyendo a la práctica, este estudio encuentra que las PYME con tamaños más grandes, plantas múltiples y cuyos propietarios tienen educación superior tienen una mayor tendencia a adoptar las TIC de forma temprana.

La continua evolución de internet como una herramienta principal de comunicación ha proporcionado nuevas aplicaciones para el remitente y el receptor, tales como correo electrónico, blogs, foros y protocolos de voz. Muchas de estas aplicaciones de comunicación basadas en internet han demostrado ser muy populares entre los grupos de consumidores. Wong et al. (2011) mediante el examen del patrón de difusión del correo electrónico, buscaron determinar cuándo este tipo de comunicación alcanzará su pico de adopción. Los autores proponen que las estimaciones de parámetros del modelo Bass para el correo electrónico pueden servir como una aproximación de las estimaciones de parámetros para otros productos dentro del dominio de aplicaciones de comunicación similares basadas en internet. Este estudio se basó en diez años de datos de difusión. El modelo Bass reveló que el efecto de imitación es mayor que el efecto de innovación en la difusión de tecnologías de comunicación similares basadas en Internet. También descubrieron que, para esas 
tecnologías, el pico de la curva de adopción no acumulativa se puede esperar en 5.4 años después de su lanzamiento inicial.

En el caso de la literatura científica podríamos sustentar que la difusión se produce no por medio de la comunicación de masas, sino por la literatura especializada publicada en las revistas académicas especializadas que circulan en las bibliotecas y servicios de información y documentación. Esta circulación es reforzada por los servicios de alerta informativa, cursos especiales proporcionados por los bibliotecarios para familiarizar a los investigadores con las bases de datos bibliográficos especializadas y los cursos especiales llamados "formación de usuarios". La comunicación interpersonal sería básicamente aquella proporcionada por los docentes en las escuelas y programas de formación de bibliotecarios, documentalistas y científicos de la información ofrecidas por las universidades de diferentes tipos y niveles. También los eventuales contactos establecidos por los investigadores y estudiantes en congresos, mesas redondas, workshops, etc.

Una vez introducida la innovación (por ejemplo, las técnicas bibliométricas) en un campo determinado, después de cierto tiempo, el número de innovadores comienza a decrecer, porque existe menos potencial de innovación. Esto genera que los imitadores aumenten, debido al efecto de la comunicación informal boca a boca. Más imitadores entran al mercado de las competencias científicas, entonces es mayor la adopción de la nueva línea de investigación de ese campo científico y la cantidad de agentes adoptantes comienza a crecer, pero crece solo hasta cierto punto (el nivel de saturación), pues, cuando llega a su "punto máximo" comenzará a decrecer. En cierto sentido, el modelo de Bass es similar a los modelos epidemiológicos o de contagio, los cuales describen la propagación de una enfermedad en una población, debido al contacto con personas infectadas y por eso proporciona una estructura conceptualmente atractiva y matemáticamente elegante para explicar cómo una nueva tecnología o producto se difunde, por medio de una población específica. La Figura 1 muestra la estructura conceptual del modelo de Bass, presenta la relación entre los nuevos adoptantes innovadores e imitadores, relación en el tiempo y pensando en el despliegue y la saturación del campo en exploración. Como "teoría de la comunicación", el principal enfoque está centrado en los canales de comunicación. Los canales de comunicación son los medios por los cuales la información acerca de una innovación se transmite en un sistema social. Estos medios consisten, tanto de los medios de comunicación de masas como de los canales de comunicación interpersonales. Los miembros individuales de un sistema social tienen diferentes tendencias de confianza en los medios de comunicación o los canales interpersonales cuando buscan información sobre una innovación. La comunicación interpersonal, incluye las observaciones no verbales, las cuales son influencias importantes que se ejercen sobre la velocidad y la forma del proceso de difusión en un sistema social. La más eficaz será siempre la docencia. 


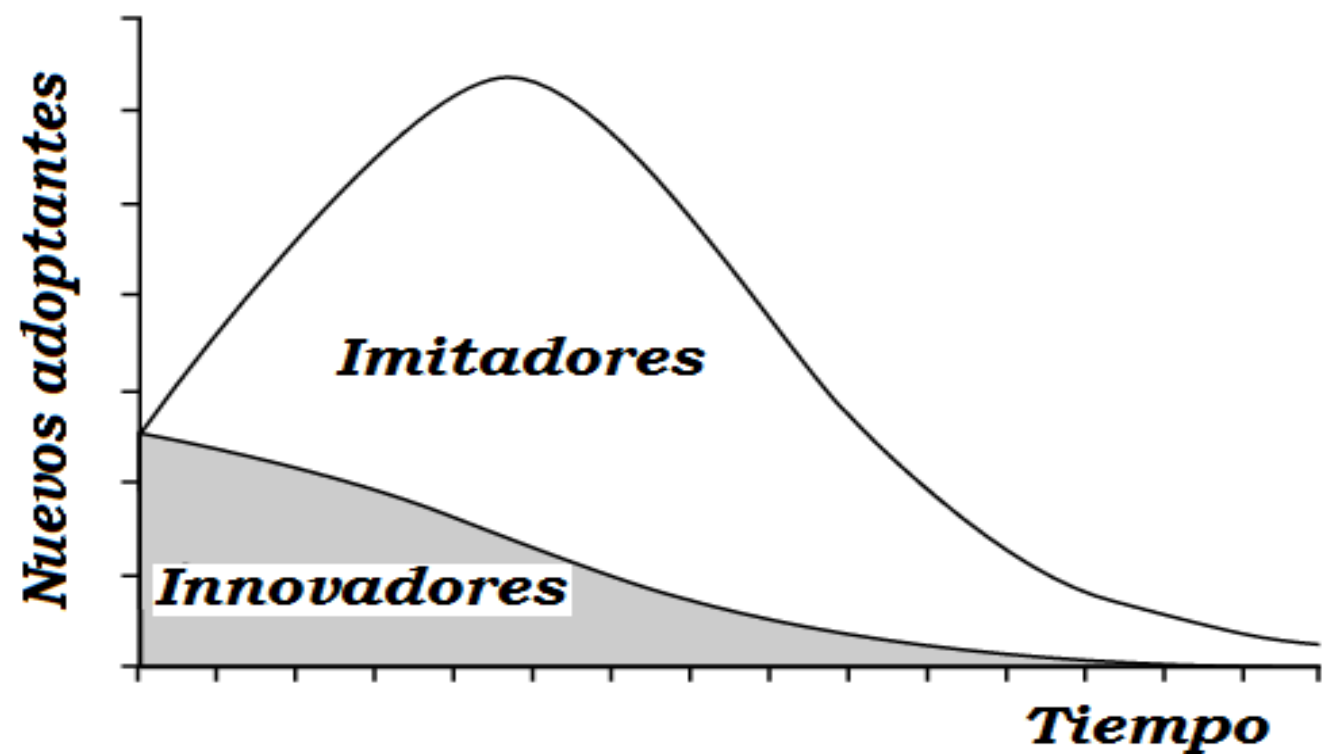

Figura 1: estructura conceptual de modelo de Bass

La difusión de las ideas científicas no es muy diferente a como se difunden las innovaciones o las nuevas tecnologías. Estas se producen, por medio de los servicios de alerta de los centros de documentación y bibliotecas, las citas en documentos, los intercambios de opiniones en eventos especializados como congresos, mesas redondas, jornadas de trabajo y aun noticias que corren de boca en boca entre investigadores que están trabajando en el mismo asunto o aquellos que tienen intereses similares, también la docencia y la enseñanza en campos científicos específicos. Por lo tanto, la adopción de las líneas de investigación en ciertos campos científicos puede mostrar comportamientos muy similares a los propuestos por el modelo de Bass (1969). Más aún, la publicación de documentos que se producen dentro de un campo científico que no deja de ser un mercado de competencias y luchas estratégicas inconscientes por la dominación y la hegemonía de la "autoridad científica" (BOURDIEU, 1983) que pueden servir como una muestra de estas innovaciones. La ciencia académica es una categoría sociológica, no porque este estructurado organizacionalmente en términos de universidades, sino porque está socialmente estructurado en términos de disciplinas. Los investigadores académicos se localizan ellos mismos y su trabajo en un mapa de fino grano de innumerables especialidades. El número de distintos campos de la ciencia y la erudición se cuentan por miles, todo cuidadosamente diferenciado en la mente de sus devotos. Estos no son solo sitios de investigación temporales, para entrar casualmente según lo requiera la ocasión. Una gran disciplina es una institución bien definida, delineada por una variedad de prácticas sociales intangibles, pero efectivas. La membresía de tal tribu no es solo una cuestión de 
preferencia personal ocasional: es un privilegio ganado por el laborioso aprendizaje y mantenido por la dedicación de toda una vida (ZIMAN, 1998).

El campo científico es el lugar de una lucha competitiva que tiene por desafío específico el monopolio de la autoridad científica, el monopolio de la competencia científica que es socialmente reconocida a un agente determinado, entendida en el sentido de capacidad de hablar e intervenir legítimamente en materia de ciencia. La autoridad científica es una especie particular de capital que puede ser acumulado, transmitido e incluso reconvertido en otras especies bajo ciertas condiciones. La literatura publicada explicita esa competencia y juega así su parte en este mercado de competencias como transmisor de innovaciones y como visualizador de la autoridad científica.

\section{Material y métodos}

Como unidades de análisis fueron tomados cada uno de los artículos publicados en revistas académicas, capítulos de libros y trabajos presentados en congresos que trataron algunos de los aspectos de los estudios métricos (bibliometría, cienciometría, informetría, etc.) o las aplicaciones de estas técnicas en una disciplina o subcampo determinado en el Brasil o por brasileros que publicaron documentos sobre este asunto fuera del Brasil. Se excluyeron los autores de libros, tesis, monografías y literatura gris por dos razones. Primero porque los libros comienzan como artículos publicados en revistas especializadas; y segundo porque las tesis, monografías y literatura gris no son indexadas en muchas de las bases de datos bibliográficas consultadas para esta investigación. El periodo cubierto va desde los primeros trabajos publicados a inicios de la década de los 70 s hasta diciembre del 2018.

Para recolectar los datos fueron efectuadas búsquedas usando los términos listados en el Anexo A en sus diferentes acepciones idiomáticas (inglés, francés, alemán, portugués, español, etc.) y en múltiples combinaciones booleanas en los títulos, palabras clave y resúmenes de las bases de datos bibliográficas y portales de bibliotecas listadas en el Anexo B. Las referencias identificadas fueron después exportadas a EndNote X8 para la elaboración de una base de datos sobre el asunto. Posteriormente, fue realizada una minuciosa lectura de cada uno de los documentos identificados en la búsqueda, dedicando especial atención a cada referencia efectuada en el documento leído. Después cada referencia relativa al asunto de investigación era confrontada con la base de datos e incorporada, si no había sido identificada en la búsqueda anterior. Lógicamente las referencias duplicadas fueron eliminadas, manteniéndose solamente una referencia no repetida. La identificación de la pertinencia del documento fue una tarea ardua y difícil, porque con mucha frecuencia las palabras clave fueron insuficientes y a veces falsos indicativos. Para identificar a los autores brasileños se analizó la afiliación institucional de cada autor en cada referencia recuperada. 
Muchas veces fue necesario consultar la Plataforma Lattes para realizar esta verificación o buscar los "currículo vitae" de los autores, y en no pocas situaciones, se realizó minería de textos y consultas individuales vía correo electrónico. La nacionalidad y la ligación institucional fueron también indagadas, por medio de mensajes personales enviados al correo electrónico del investigador identificado. Con toda esa estrategia y la lectura minuciosa de muchos de los artículos identificados fue producida la base de datos (que sirve de base al objeto de esta investigación) que contiene referencias de artículos de revistas, capítulos de libros, comunicaciones presentadas en congresos, una que otra nota editorial y cartas enviadas a los editores de las revistas académicas. Esta base de datos especializada ha estado en construcción permanente por un periodo de más de siete años.

Para medir los datos de esta literatura se utilizó el modelo de Bass (1969) que se origina de una hipótesis comportamental, es decir, supone que durante el proceso de difusión de una nueva innovación, dos tipos de usuarios determinarán cómo crecerá la demanda. El primer tipo de usuario es aquel que decide adoptar la innovación de manera independiente, es decir, no recibe influencia directa de otros adoptantes, pero pueden ser influenciado por otros medios como la comunicación de masas. Estos individuos son los llamados "innovadores", son los primeros adoptantes. El segundo tipo corresponde a los potenciales adoptantes que son influenciados por la presión social del medio y también son susceptibles de recibir las influencias de los otros productores que ya adoptaron la innovación o que ya adquirieron la técnica. Estos son los llamados "imitadores".

Esta hipótesis de comportamiento del investigador/usuario puede ser transcrita directamente en un modelo matemático. Considérese que $S(t)$ sea el total acumulado en el tiempo t de investigadores que ya han producido un artículo determinado (primera producción) en un campo del conocimiento potencial. El modelo de Bass en su forma más resumida afirma que la “presión por la adopción, digamos, $\mathrm{P}(\mathrm{t})$, corresponde a la probabilidad de adquisición en el tiempo, por un productor aleatorio, es una función lineal dela proporción de investigadores que ya adoptaron (adquirieron) las técnicas bibliométricas, es decir:

$$
P(t)=p+q\left(\frac{S(t)}{m}\right)
$$

La ecuación (1) es la premisa fundamental del modelo de Bass. Si esta premisa es verdadera, entonces el parámetro $\mathbf{p}$ corresponderá a la tendencia con que un individuo adopta una nueva técnica bibliométrica, generalmente estimulado por el proceso de comunicación social (HORSKY; SIMON, 1983, KALISH; LILIEN, 1986, KALISH, 1985). Este parámetro es llamado en la literatura de "tasa de innovación". 
El parámetro q está vinculado a una tendencia de adopción generada por "contagio social". Si se observa el segundo término de la ecuación (1), este crecerá en la medida en que aumenta la proporción de agentes que adoptan la innovación, $(\mathrm{S}(\mathrm{t}) / \mathrm{m})$, y este crecimiento es proporcional al parámetro q. Por lo tanto, el parámetro q corresponde a la fuerza que se recibe por contagio, por eso en la literatura es denominada de "tasa de imitación".

Puesto que $S(t)$ es el total acumulado en el tiempo $t$ de los investigadores que ya han adquirido la técnica bibliométrica, $\mathrm{dS}(\mathrm{t}) / \mathrm{dt}$ será la tasa de crecimiento de los nuevos productores en cada momento del tiempo t. Por lo tanto, la proporción de individuos en el tiempo t que ya adoptaron la técnica en relación con aquellos que aún no la han adoptado, será determinado por:

$$
h(t)=\left(\frac{1}{m-S(t)}\right) \times \frac{d}{d t} S(t)
$$

donde $\mathbf{m}$ es el número total de potenciales adoptantes de la innovación.

Combinando la ecuación (1) con la ecuación (2) y haciendo que $U(t)=(m-S(t))$ sea el número de adoptantes potenciales que aun podrán adquirir la innovación, se obtiene el modelo de Bass en su forma de ecuación diferencial:

$$
\frac{d}{d t} S(t)=p \times U(t)+q \times U(t) \times \frac{S(t)}{m}
$$

La curva $S(t)$ obtenida por la solución de la ecuación (3) mostrará un patrón de crecimiento logístico, mostrará también un punto de saturación en $\mathrm{S}(\mathrm{t})=\mathrm{m}$, comenzando su declive como se ejemplifica en la Figura 2. 


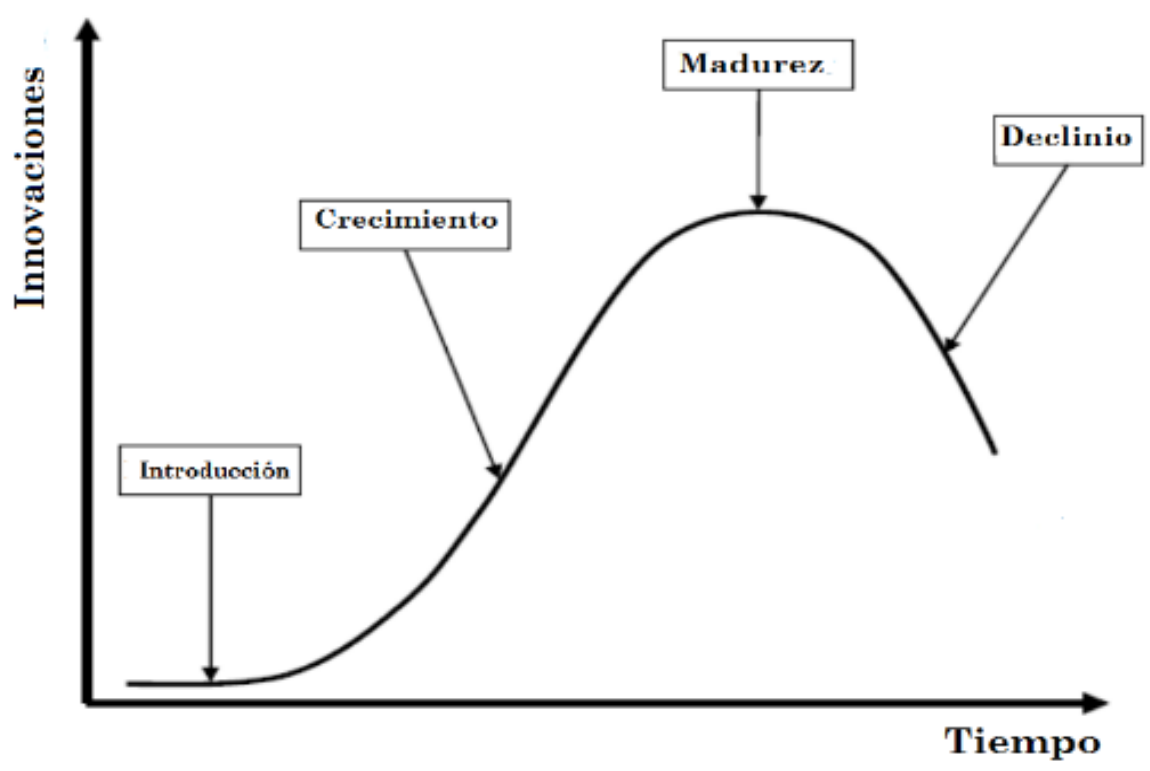

Figura 2. Curva teórica de la difusión de innovaciones

Para estimar los datos se usó el método del mínimo cuadrado no lineal que conduce a un mejor ajuste del modelo además de ser de fácil implementación. Para eso se usó la función de regresión no lineal del software estadístico R (R Core Team, 2017). La destreza y ductilidad de $R$ provienen de las diversas funciones y objetos que se proporcionan para su uso que son facilitados por una variedad de paquetes individuales. Para usar el contenido de un paquete, este debe ponerse a disposición de $R$, y luego cargarlo en una sesión de $R$ especifica. Sin embargo, algunos paquetes siempre están disponibles en $\mathrm{R}$ al inicio de la sesión y se cargan automáticamente al inicio del trabajo.

\section{Resultados}

La Tabla 1 muestra el desglose por años de los 6170 documentos encontrados desde 1973, cuando se publicaron los primeros trabajos hasta diciembre de 2018, un largo período de 45 años. El volumen de documentos fue dividido y organizado por períodos de 3 años con la intención de mostrar mejor el crecimiento de la literatura publicada. Sin embargo, para la aplicación del modelo de Bass se usaron los datos no agrupados. Aquí se muestra los datos agrupados solamente para evitar una larga lista de datos de dos páginas. 
Tabla 1. Documentos publicados por trienios, 1971-2018

\begin{tabular}{lcc} 
Trienios & $\begin{array}{c}\text { Número de } \\
\text { documentos }\end{array}$ & Porcentaje \\
\hline $1971-1973$ & 7 & 0.11 \\
$1974-1976$ & 20 & 0.33 \\
$1977-1979$ & 22 & 0.36 \\
$1980-1982$ & 23 & 0.37 \\
$1983-1985$ & 22 & 0.36 \\
$1986-1988$ & 29 & 0.47 \\
$1989-1991$ & 16 & 0.26 \\
$1992-1994$ & 21 & 0.34 \\
$1995-1997$ & 36 & 0.58 \\
$1998-2000$ & 80 & 1.30 \\
$2001-2003$ & 134 & 2.18 \\
$2004-2006$ & 258 & 4.19 \\
$2007-2009$ & 562 & 9.13 \\
$2010-2012$ & 1087 & 17.66 \\
$2013-2015$ & 1799 & 29.23 \\
$2016-2018$ & 2018 & 32.79
\end{tabular}

La Tabla 2 muestra los resultados de los parámetros estimados de la curva de difusión de Bass para la literatura publicada sobre los estudios métricos en el Brasil desde 1973 hasta 2018.

Tabla 2: Parámetros estimados según el modelo de Bass

\begin{tabular}{cllll} 
Parámetros & Estimados & error patrón & valor $\mathrm{t}$ & $\operatorname{Pr}(>/ \mathrm{t} /)$ \\
\hline $\mathrm{m}$ & 165450.09322 & 19938.84591 & 8.298 & 0.00000000018 \\
$\mathrm{p}$ & 0.0000024126 & 0.000000394 & 6.110 & 0.00000025271 \\
$\mathrm{q}$ & 0.2208777087 & 0.007028084 & 31.428 & $<0.0000000000000002$
\end{tabular}

Se usó el método de los mínimos cuadrados no lineales que conduce a un mejor ajuste del modelo, además de ser de fácil implementación. Los parámetros estimados revelan que el efecto de la innovación $(p=0.0000024)$ es estadísticamente significante ( $p$-value0.00000000018<0.05) en la predicción de la adopción y producción de la literatura sobre la bibliometría brasilera. También el efecto de la imitación $(q=0.2209)$ es estadísticamente significante $(p$-value0 0000000000000002 $<0.05$ ) en la predicción de la adopción y producción en la literatura sobre la bibliometría brasilera, ambos con 43 grados de libertad y con un error residual de 78.84 .

La Figura 3 muestra la línea de regresión de los valores observados y estimados de este modelo que se ajustan adecuadamente a los datos de la bibliometría brasilera, los coeficientes muestran signos positivos y son estadísticamente significativos al nivel del $5 \%$. El trazado de los 
datos y las predicciones son consistentes con la teoría del modelo de Bass. El coeficiente de innovación $p(0.0000024126 \%)$ captura la influencia sobre los potenciales adoptantes considerados como independientes en relación con la opinión de los adoptantes existentes al momento de la adopción.

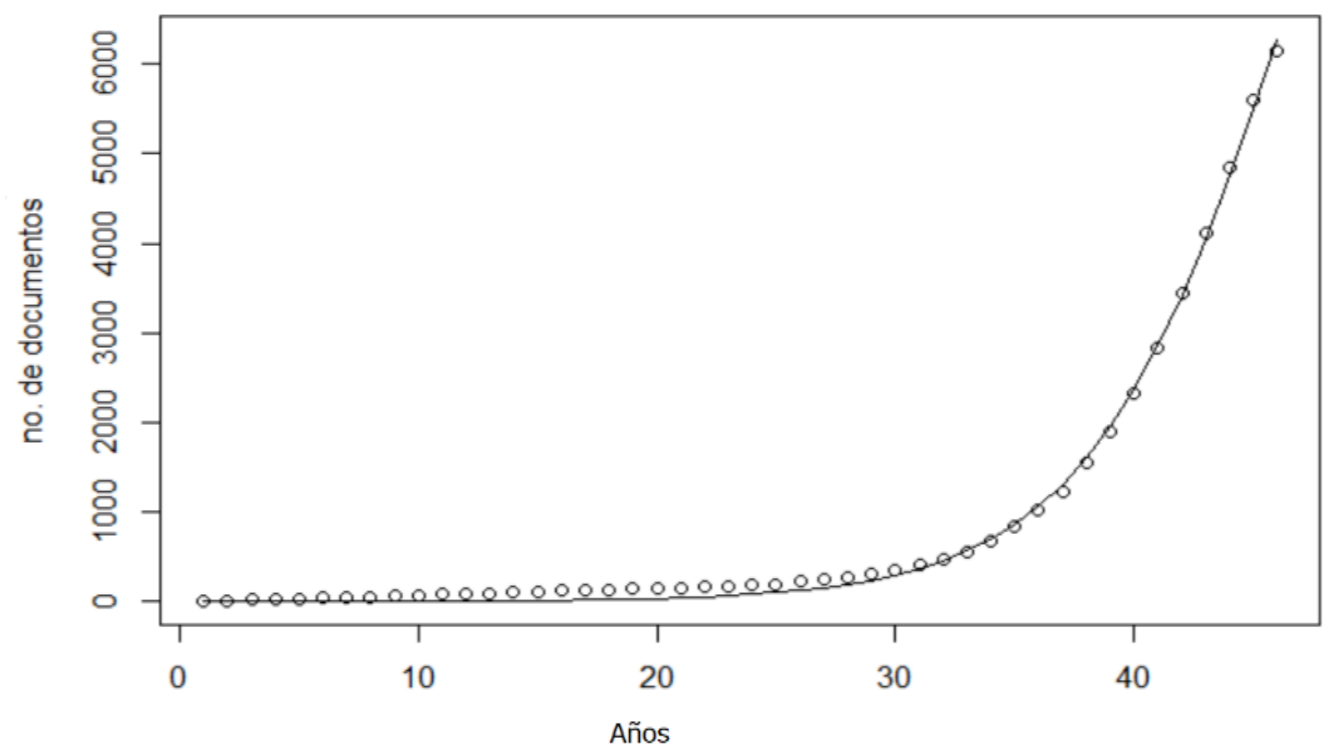

Figura 3. Ajuste del modelo de Bass a las publicaciones sobre bibliometría brasilera, 1973-2018

Esto significa que la influencia capturada por el coeficiente $\mathbf{p}$ no procede de la comunicación interpersonal tradicional (comunicación oral boca a boca), sino que procede del acceso que el investigador tiene a la literatura sobre bibliometría brasilera (acceso y uso a bases de datos, bibliotecas, servicios de alerta, servicios de diseminación selectiva de la información, cursos de entrenamiento sobre búsqueda de información ofrecidos en las bibliotecas, etc.) y a la habilidad personal que los investigadores tienen para obtener información recurriendo a otros canales que no sean los de la comunicación informal boca a boca (por ejemplo, notas en las revistas, noticias en la TV, citas en los documentos leídos, etc.). Cuanto mayor sea el tiempo que los investigadores estén expuestos a este tipo de comunicación de informaciones, mayor será la propensión para obtener más información sobre este asunto, haciendo que la penetración de la literatura aumente conforme aumentan los años recorridos desde la publicación del primer documento sobre este asunto hasta el último documento publicado y recuperado.

Igualmente, investigadores con mayor grado académico (mayor nivel educativo) son más propensos a buscar más información, por lo tanto a publicar también más información. Estos investigadores son los más inclinados a utilizar otras fuentes y canales de comunicación para encontrar información y evaluar la calidad de la literatura publicada. Sin embargo, en la producción 
de documentos sobre la bibliometría brasilera la influencia que los investigadores reciben de los medios de comunicación indicados anteriormente es de apenas $0.0002 \%$, es decir, de la pura lectura de otros documentos sobre este mismo asunto en los sistemas de información. Esta influencia que procede de los sistemas de información es insignificante, bien por debajo del $1 \%$.

El "coeficiente de imitación" (22\%) mide la influencia que un grupo de adoptantes ejerce sobre las decisiones de adopción de otros potenciales adoptantes. En otras palabras, mide la influencia que un conjunto de investigadores que publicaron literatura sobre la bibliometría brasilera ejerce sobre las decisiones de publicar sobre el mismo asunto por aquellos investigadores que aún no publicaron ningún documento sobre la bibliometría brasilera. El coeficiente de imitación captura la efectividad de la comunicación interpersonal como elemento impulsor de las decisiones de publicar sobre este mismo asunto. Este coeficiente está básicamente relacionado con la homogeneidad cultural y educativa de los investigadores y su capacidad de persuasión. Por lo tanto, será mayor en los campos del conocimiento donde la capacidad de persuasión de las recomendaciones informales boca a boca sea más eficiente entre los posibles adoptantes. Esta capacidad aumenta cuando entre los adoptantes existe mayor familiaridad y satisfacción con el asunto investigado, en este caso, la bibliometría brasilera. Representa el nivel de familiaridad y satisfacción de los investigadores en relación al número de años que el asunto bibliometría brasilera ha estado siendo publicado en el campo científico de las ciencias bibliotecológicas y afines, es decir, si se han publicado documentos sobre este asunto por varios años, hay más posibilidades que errores y deficiencias encontrados en el proceso de investigación hayan sido corregidas y que ya exista un largo camino de aprendizaje y consolidación de la disciplina, por lo tanto, las noticias de esos "sucesos académicos" atraerán más investigadores.

La Figuras 4 muestra la tasa de adopción, ésta indica que la literatura sobre la bibliometría brasilera estará llegando a su punto más elevado (el tope) más o menos por el año 2030. A partir de este punto de tope comenzará a declinar en publicaciones hasta llegar a desaparecer (la obsolescencia) o renovarse. 


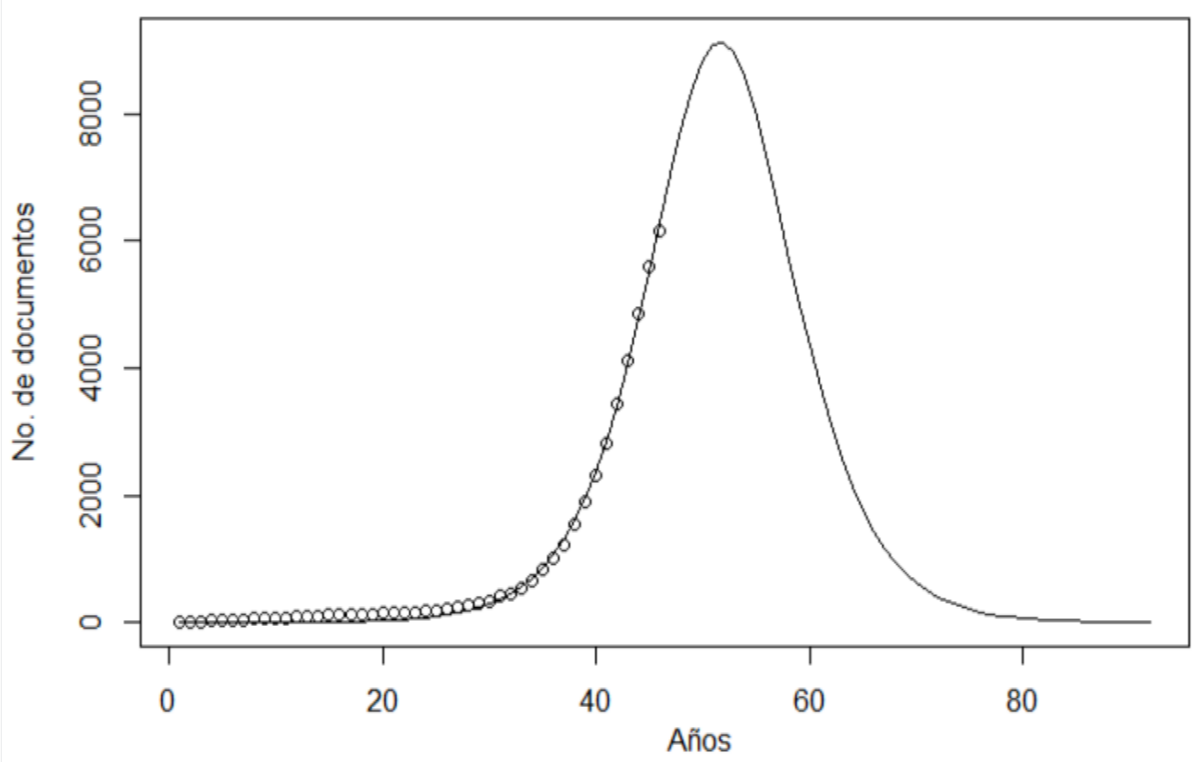

Figura 4. Pique de la tasa de adopción

Sin embargo, el parámetro $\mathbf{m}$ muestra el punto de saturación del campo, en este caso de las publicaciones sobre la bibliometría brasilera. El modelo de Bass estima que cuando en el campo se hayan producido 165,450 documentos, este asunto habrá alcanzado su madurez y el campo comenzará a desaparecer o se renovará para pasar a un estado de investigaciones diferentes. La Figura 5, muestra que la tasa de penetración de los autores de la literatura sobre las metrías en el Brasil es larga y que alcanzará su punto de obsolescencia en 630 años, es decir más o menos por el año 2500. Esto si el ritmo de entrada de nuevos autores al campo es asegurado y la producción de documentos siga con el mismo ritmo de crecimiento mostrado hasta ahora.

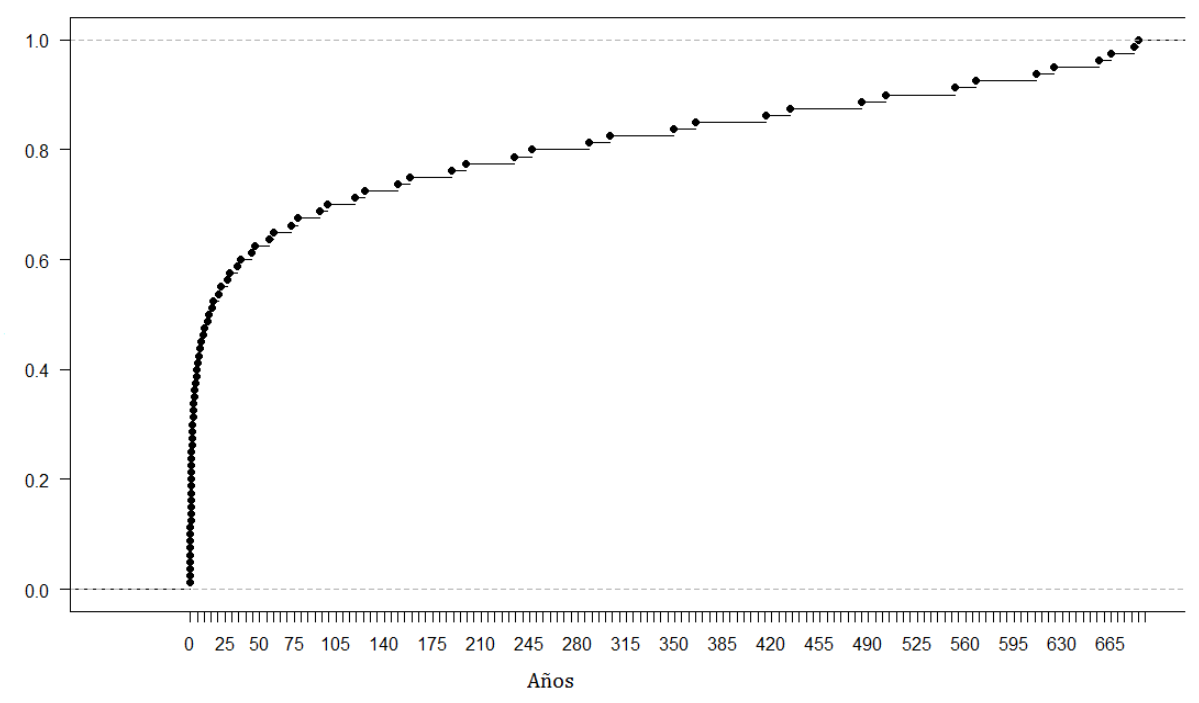

Figura 5. Pique de la tasa de saturación 


\section{Conclusiones}

El modelo de Bass asume que la forma tradicional en forma de $S$ invertida de las curvas de adopción de las nuevas innovaciones proviene de una mezcla de influencias internas y externas. Las influencias internas son las que surgen de las interacciones dentro del sistema social, como la comunicación informal boca a boca. Las influencias externas provienen de agentes de cambio; por ejemplo, mensajes publicitarios que están fuera del sistema social. La combinación de estos dos factores describe la forma de adopción de un nuevo producto en el mercado.

Esta investigación trasladó el modelo de Bass al proceso de producción de literatura científica como el producto de un proceso de investigación especializado que se genera también en un campo especializado. La idea es explicar la forma en la que la literatura académica sobre la bibliometría brasilera comienza a ser publicada, cómo inicialmente aparece una literatura "innovadora" (el primer o primeros documentos publicados) y cómo luego aparecen nuevos adoptantes o imitadores (los segundos, terceros, cuartos, etc. documentos publicados) de esa nueva línea de investigación. De manera similar al proceso de adopción de nuevas innovaciones, también la literatura publicada parece estar sujeta a los dos mecanismos de adopción de las innovaciones. Primero, los mecanismos de influencias internas que surgen de las interacciones dentro del sistema de comunicación de la información científica, como los mecanismos de diseminación selectiva de la información, los servicios de alertas en las bibliotecas, los cursos ofrecidos sobre el uso de bases de datos bibliográficos y los esfuerzos de alfabetización informacional, y otros servicios similares impartidos en los sistemas de información, documentación y bibliotecas. Sin embargo, estos esfuerzos de educación de usuarios para la diseminación de la información parecen tener poco efecto sobre la apertura de nuevas líneas de investigación o sobre la generación de nueva literatura científica. Los efectos provenientes de este esfuerzo solo son responsables de menos del $1 \%$ de los procesos de generación de nuevos documentos publicados.

Segundo, las influencias provenientes de la capacidad de disuasión de los autores que ya adquirieron una autoridad científica en el campo y los mecanismos de difusión informales 'boca a boca" parecen tener mayor efecto sobre la nueva generación de documentos y la entrada de nuevos investigadores en el campo. Estos son responsables de $22 \%$ de los efectos en el caso de la literatura publicada en la bibliometría brasilera. Se sospecha que esta capacidad de disuasión de los autores orientada a la producción de literatura sobre la bibliometría brasilera parece ser ejercida por los profesores y docentes que enseñan las técnicas y modelos bibliométricos en los programas de formación educativa en Bibliotecología y ciencia de la información brasileras, especialmente los cursos de postgrado. 
Los resultados de esta investigación muestran que el modelo de Bass es capaz de modelar la generación de nueva literatura, así como de estimar el crecimiento y acumulación de la literatura hasta llegar a un punto de saturación que indica su madurez, comienza a descender, se inicia su obsolescencia. Es en este punto, en palabras de Engels (1932) que la ciencia siempre da un salto cualitativo, esta se renueva. La obsolescencia de las tecnologías, la desaparición de las profesiones, son las consecuencias inevitables de las constantes innovaciones tecnológicas, pero abren paso a la aplicación y proliferación de nuevas técnicas. El progreso tecnológico y la innovación se aceleran y se renuevan, se transforman, continúan desarrollándose.

Sin embargo, mayores investigaciones podrían producir resultados diferentes en otros campos. Por lo tanto, es aconsejable realizar mayores investigaciones para validar y generalizar el resultado de la presente investigación

\section{Bibliografia}

BASS, F.M. A new product growth model for consumer durables. Management Science, v. 13, n. 5, p. 215-227, 1969.

BOURDIEU, P. O campo científico. In: BOURDIEU, Pierre. Sociologia. São Paulo: Ática, 1983. p. 122155.

ENGELS, F. Socialism: utopian and scientific. London: Allen \& Unwin, 1932.

FOURT, L.; WOODLOCK, J. Early prediction of market success for new grocery products. The Journal of Marketing, v.25, n.2, p.31-38, 1960.

GARG, K.; PRAVEEN, S.; SHARMA, L. Bradford's law in relation to the evolution of a field: a case study of solar power research. Scientometrics, v. 27, n. 2, p. 145-156, 1993.

GARCÍA GONZÁLEZ, F. Conceptos sobre innovación: contribución al análisis PEST (Política, Economía, Sociedad, Tecnología), "Plan estratégico 2013-2020". Bogotá, Colombia, Asociación Colombiana de Facultades de Ingeniería, octubre 2012.

GARVEY, W.D. Communication: the essence of science: facilitating information Exchange among librarians, scientists, engineers and students. Oxford: Pergamon Press, 1979.

GARVEY, W.D.; GRIFFITH, B.C. Scientific communication as a social system. In: Garvey, William D., Communication: the essence of science: facilitating information Exchange among librarians, scientists, engineers and students, Oxford, Pergamon Press, 1979. p. 148-164.

GARZÓN, M. A.; IBARRA, A. Innovación empresarial, difusión, definiciones y tipología. Una revisión de literatura. Revista Dimensión Empresarial, v. 11, n. 1, p. 45-60, 2013.

HO, Y. S.; CHIU, C. H.; TSENG, T. M.; CHIU, W. T. Assessing stem cell research productivity. Scientometrics, v. 57, n. 3, p. 369-376, 2003. 
HORSKY, D. The effects of income, price and information on the diffusion of new consumer durables. Marketing Science, v. 9, n .4, p. 342-365, 1990.

HORSKY, D.; SIMON, L. S. Advertising and the diffusion of new products. Management Science, v. 1, p. 31-47. 1983.

JAIN, A.; GARG, K. C. Laser research in India: Scientometric study and model projections. Scientometrics, v. 23, n. 3, p. 395-415, 1992.

KALISH, S. A new product adoption model with price, advertising and uncertainty. Management Science, v. 31, n. 12, p. 1569-1585, 1985.

KALISH, S.; LILIEN, G. L. A market entry timing model for new technologies. Management Science, v. 32, n. 4, p. 194-205. 1986.

KAPOOR, K.H.; DWIVEDI, Y.K.; WILLIAMS, M.D.; LAL, B. An analysis of existing publications to explore the use of the diffusion of innovations theory and innovation attributes. WORLD CONGRESS ON INFORMATION AND COMMUNICATION TECHNOLOGIES. Proceedings, p. 229-234, 2011.

LESSIG, L. The future of ideas: the fate of the commons in a connected world. New York, Random House, 2001.

LOZANO MEJÍA, J. M. Los gigantes de Newton. Ciencias: revista de Difusión, v. 12, p. 30-35, 1988.

MACHADO, R. N.; PINTO, E. V. Mapeamento da produção científica em bibliometria (1990-2004). In: ENANCIB: ENCONTRO NACIONAL DE PESQUISA EM CIÊNCIA DA INFORMAÇÃO, 6., 2005, Florianópolis, SC. Anais do VI Enancib. Florianópolis: Universidade Federal de Santa Catarina, 2005.

MAHAJAN, V.; MULLER, E.; BASS, F. M. New Product Diffusion Models in Marketing: A Review and Directions for Research. Journal of Marketing, v. 54, n. 1, p. 1-26, Jan. 1990.

MANSFIELD, E. Technical change and the rate of imitation. Econometrica, v. 29, n. 4, p. 741-766, 1961.

MIN, C.; YING, D.; JIANG, L.; YI; BU, L.; PEI, E, JIANJUN, S. Innovation or imitation: The diffusion of citations. Journal of the Association for Information Science and Technology, v. 69, n. 10, p. 1271$1282,2018$.

NEWTON, I. Letter from Sir Isaac Newton to Robert Hooke. Historical Society of Pennsy/vania, February 5, 1675.

NTWOKU, H.; SOLOMON NEGASH; E.; MESO, P.ICT adoption in Cameroon SME: application of Bass diffusion model. Information Technology for Development, v. 23, n. 2, p. 296-317, 2017.

PÉREZ PULIDO, M.; TERRÓN TORRADO, M. La teoría de la difusión de la innovación y su aplicación al estudio de la adopción de recursos electrónicos por los investigadores en la Universidad de Extremadura. Revista Española de Documentación Científica, v. 27, n. 3, p. 308-329, 2004.

PIERCE, S. J. Disciplinary work and interdisciplinary areas: sociology and bibliometrics. In: BORGMAN, C.L. (Ed.). Scholarly communications and bibliometrics. Newbury Park, CA: Sage Publications, 1990. p. 46-58. 
PINHEIRO, L.V.R.; SILVA, G.S. Cartografia histórica e conceitual da bibliometria / informetria no Brasil. In: CONFERÊNCIA IBERO-AMERICANA DE PUBLICACOES ELETRONICAS NO CONTEXTO DA COMUNICACAO CIENTIFICA, II CIPECC., 2008, Rio de Janeiro, RJ. Rio de Janeiro, RJ, Brasil, 17 a 21 de novembro de 2008.

PRICE, J.D.S. The structures of publication in science and technology. In: GRUBER, W.H.; DONALD, G. (Eds.). Factors in the transfer of technology. Marquis, Cambridge, Mass.: M.I.T. Press, 1969. p. 91104

R CORE TEAM. R: A language and environment for statistical computing. R Foundation for Statistical Computing, Vienna, Austria, 2017. Disponible en: URL https://www.R-project.org/ Consultado en: 8 ago. 2019.

RESTREPO-ARANGO, C. La teoría de la difusión de las innovaciones en la cultura zenú. Ciência da Informação em Revista, v. 5, n. 3, p. 3-12, 2018.

RYAN, B.; GROSS, N.C. The diffusion of hybrid seed corn in two lowa communities. Rural Sociology, V. 8, n. 1 , p. 15,1943 .

ROGERS, E. M. Diffusion of innovations. New York: Free Press of Glencoe, 1962.

URBIZAGÁSTEGUI-ALVARADO, R. A bibliometria no Brasil. Ciência da Informação, v. 13, n. 2, p. 91105, 1984.

URBIZAGÁSTEGUI-ALVARADO, R. El modelo de Bass en la literatura sobre ArgopectenPurpuratus. Ciência da Informação, v. 46, n. 2, p. 67-83, maio/ago. 2017.

WATERMAN, A.T. Social influences and scientists. Science, New Series, v. 151, n. 3706, p. 61-64, Jan.7, 1966

WEIMANN, G. The influentials. Albany, New York, NY: State University of New York Press, 1994.

WEISS, Paul. Knowledge: a growth process. Science, New Series, 1313415, p. 1716-1719, Jun. 10, 1960.

WONG, D. H.;YAP, K. B. TURNER, B.; REXHA, N. Predicting the diffusion pattern of internet-based communication applications using bass model parameter estimates for email. Journal of Internet Business, v. 9, p. 26-50, 2011.

ZIMAN, JOHN. Basically, it's purely academic. Interdisciplinary Science Reviews, v. 23, n. 2, p. 161168, 1998.

ZIMAN, JOHN. Information, Communication, Knowledge. Nature, v. 224, p. 318-324, October 25, 1969. 
Brasil / Índice h / Elitismo / Frente de / investigación / Regla 80/20 / Obsolescencia de la literatura / Crecimiento de la literatura / Vida media / Teoría epidémica / Visibilidad / Índice de Pratt / Índice de Price / Índice de inmediatismo / Ley de Price / Indicadores bibliométricos / Indicadores cienciométricos/ Ley de Goffman / Ley de Bradford / Ley de Lotka/ Ley de Zipf/ Punto de transición / Colegios invisibles / Factor de impacto / Factor de inmediatismo / Análisis de citas / Acoplamiento bibliográfico / Co-citación/ Redes sociales / Co-autoría/ Colaboración científica / Índice de colaboración / Circulación de la colección / Núcleo básico de periódicos / Indicadores en ciencia y tecnología / Bibliometría / Cienciometría / Informetría/ Patentometría/ Arquivometría/ Biobibliometría / Webometría/ Sitometría

Netometría 
ANEXO B: Bases de Datos Bibliográficos y Portales consultadas

Library Literature\& Information Science Full Text

Library and Information Science Abstract (LISA)

Library, Information Science \& Technology Abstracts (LISTA)

Plataforma Lattes

$B R A P C I$

$\mathrm{LICl}$ (IBICT)

PERI: Base de Dados de Periódicos (UFMG)

Biblioteca Virtual em Saúde

SPELL: ScientificPeriodicalsElectronic Library

DEDALUS: Banco de dados Bibliográficos da USP

Web of Science

Scopus

JSTOR

Agrícola

Biosis

CAB Abstracts

Medline

Anthropological Literature

Anthropological Index

Anthropology Plus

WorldCat

$\mathrm{HAPI}$

ArticleFirst

Science Citation Expanded Index

Google

Google Scholar

ISOC

ICYT

Dialnet

INFOBILA de México

Periodica

Redalyc

Scielo Brasil

Scielo México

Scielo Venezuela

Scielo Colombia

Scielo Chile

Scielo Argentina

Scielo Bolivia

Portal del Ricyt

Biblioteca Virtual en Salud del Brasil

Biblioteca Virtual de la Universidad de São Paulo

y otras 520 bases de datos bibliográficas existentes en la biblioteca de ciencias de la Universidad de California en Riverside. 